\section{Nanoscale Technique Prints Metal Films with Reusable Stamps and Tailored Surface Chemistry}

Traditional nanolithographic techniques such as electron-beam or deep-UV nanolithography are limited to patterning small regions of specialized materials on rigid, ultraflat inorganic substrates. These restrictions are unacceptable for nascent applications in fields such as plastic electronics and biotechnology. Consequently, researchers are aggressively investigating alternative "soft lithography" methods, such as near-field phase-shift lithography, microcontact printing, and dip-pen lithography. In the July 15 issue of Applied Physics Letters, Y.-L. Loo and co-workers at Lucent Technologies described a nanotransfer printing (nTP) technique that is used to transfer metal films with features as small as $100 \mathrm{~nm}$ from the raised regions of a stamp onto substrates. This is reminiscent of woodcut printing, but on the nanometer scale. The high-resolution method is fast, can cover large areas, provides good adhesion, and is conducted under ambient conditions. Moreover, unlike its competing technologies, this soft lithography method is a one-step process and purely additive: It does not require any etching, resists, or postpatterning deposition.

The scientists produced rigid stamps by conventional patterning and etching of hard substrates such as glass or GaAs and created flexible stamps by casting a prepolymer of poly(dimethysiloxane) (PDMS) in a patterned resist mold. They "inked" the stamp simply by evaporating the desired metal. Substrates can also be either rigid or conformal, and materials have included silicon dioxide and PDMS.

Intimate contact between the film and the substrate transfers the metal film through a common condensation reaction between hydroxyl $(-\mathrm{OH})$ groups present on both surfaces. In order to accumulate the hydroxyl groups, the researchers applied either oxygen plasma or UV radiation to the native oxides.

This surface-chemistry restriction on the materials is not severe. Thus far, the researchers have successfully used nTP to print films of aluminum, whose surface spontaneously oxidizes, and, surprisingly, gold, after dusting it with titanium to obtain the requisite oxide layer.

"By exploiting other interfacial chemistries," Loo said, "nanotransfer printing may be suitable for patterning a wide range of single- and multilayer conducting, dielectric, and semiconducting films."

To appraise nTP's potential application to plastic electronics, the scientists patterned
$\mathrm{Au} / \mathrm{Ti}$ contacts and interconnects for organic transistors and complementary inverter circuits. The devices performed as well as top-contact devices fabricated with conventional shadow-mask gold electrodes.

RICHARD N. LOUIE

\section{Diblock Copolypeptide Amphiphiles Form Rapidly Recovering Hydrogels}

Protein-based hydrogels are used in a number of applications including drug delivery, tissue replacement, and cosmetics. Researchers at the University of California, Santa Barbara and the University of Delaware, Newark have synthesized diblock copolypeptides, composed of hydrophobic and hydrophilic domains, that form rigid hydrogels at very low concentrations $(0.25-2.0 w t \%)$. Gelation depends on the amphiphilic nature of the polypeptides as well as on the particular conformation of the hydrophobic domain. The porosity, thermal stability, and rapid recovery from network disruption make these materials attractive for a variety of biotechnological and biomedical applications.

As reported in a letter to Nature in the May 23 issue, a team led by University of California researcher Timothy J. Deming employed metal-mediated $\alpha$-amino-acid $\mathrm{N}$-carboxyanhydride polymerizations to control both the polypeptide chain lengths and composition of the amphiphiles. Poly(L-lysinen-HBr) or poly(L-glutamate sodium salt), both highly charged polyelectrolytes at neutral $\mathrm{pH}$, make up the hydrophilic blocks. The hydrophobic blocks are composed of poly(L-leucine) or poly(L-valine), which form rodlike $\alpha$-helices and crystalline $\beta$-sheets, respectively. Copolypeptides of identical compositions to those that formed hydrogels but with random sequences did not form hydrogels at all.

Rheological measurements were made to investigate the nature of gel formation, the absolute strength of the gels, and the dependence of gelation on molecular parameters. The research team found that the hydrogels were one or more orders of magnitude stronger than an aqueous gelatin gel of the same weight percent. No visible thinning was observed up to $90^{\circ} \mathrm{C}$; most protein gels generally start to dissolve at $60^{\circ} \mathrm{C}$. No gelation occurs when the secondary structure $(\alpha$-helix or $\beta$-strand) of the hydrophobic domain is precluded, either by incorporating a racemic mixture of amino-acid residues or simply by decreasing the degree of polymerization. This suggests that the self-assembly process is conformationspecific, similar to protein assembly, the research team said. The addition of saltfor example, $25 \mathrm{mmol} \mathrm{NaCl}$-weakened the gels, presumably through chargescreening of the polyelectrolytes. After large-amplitude oscillations were applied to break down the gel structure, recovery was probed by measuring the storage and loss moduli in the linear, smalldeformation regime as a function of time. After about $10 \mathrm{~s}$, the hydrogels recovered $80-90 \%$ of their strength, followed by a slower reorganization, after which the full initial storage modulus was restored. The researchers attributed this rapid recovery to the relatively low molecular mass of the copolypeptides, which enables them to reorganize quickly.

Gel morphology was visualized using cryogenic transmission electron microscopy (CTEM) and laser scanning confocal microscopy. Hydrogel formation appeared to coincide with the intergrowth of microscopically phase-separated domains of $1-25 \mu \mathrm{m}$ in size. CTEM visualization of the vitrified gel domains revealed a complex membrane assembly responsible for network formation at the nanoscale level. Gel porosity was verified with microrheological experiments, in which the Brownian motion of micrometer-sized tracer particles dispersed in the sample was measured. In a (L-lysine-HBr) ${ }_{160}$ (L-leucine) $)_{40}$ gel, $0.5-\mu \mathrm{m}$ tracer particles diffused freely, while the motion of $1.0-\mu \mathrm{m}$ particles was restricted, which is taken as a measure of the lower size limit of the voids. This microscopic heterogeneity persisted after prolonged standing, heating, centrifugation, and bulk shear.

The researchers said that the very low mass fractions in these polypeptide gels, together with their microporous structure and recovery properties, may allow them "to fill...[a] unique niche between conventional polymer and surfactant hydrogels." These gels also possess the "advantageous features of proteins, such as degradability and functionality, which [make] them attractive for biomedical applications," said the researchers.

SteVEN TROHALAKI

\section{Anode-Supported SOFC Performs Well at Intermediate Operating Temperatures with a Lanthanum Ferrite-Based Cathode}

A group of researchers at Pacific Northwest National Laboratory in Washington has fabricated a strontium-doped lanthanum ferrite cathode that has a comparable performance with typical cathode materials, but at intermediate temperatures of $650^{\circ} \mathrm{C}$ to $750^{\circ} \mathrm{C}$. A typical solidoxide fuel cell (SOFC) configuration 